\title{
Long's equation in terrain following coordinates
}

\author{
M. Humi \\ Department of Mathematical Sciences, Worcester Polytechnic Institute, 100 Institute Road, Worcester, MA 01609, USA
}

Received: 10 February 2009 - Revised: 27 July 2009 - Accepted: 27 July 2009 - Published: 7 August 2009

\begin{abstract}
Long's equation describes two dimensional stratified atmospheric flow over terrain which is represented by the geometry of the domain. The solutions of this equation over simple topography were investigated analytically and numerically by many authors. In this paper we derive a new terrain following formulation of this equation which incorporates the terrain as part of the differential equation rather than the geometry of the domain. This new formulation enables us to compute analytically steady state gravity wave patterns over complex topography in some limiting cases of the parameters that appear in this equation.
\end{abstract}

\section{Introduction}

Long's equation (Long, 1952, 1953, 1955, 1959) models the flow of stratified incompressible fluid in two dimensions over terrain. When the base state of the flow (that is the unperturbed flow field far upstream) is without shear the numerical solutions (in the form of steady lee waves) of this equation over simple topography (i.e. one hill) were studied by many authors (Drazin, 1961, 1967; Durran, 1992; Lily, 1979; Peltier, 1983; Smith, 1980, 1989; Yih, 1967; Davis, 1999). The most common approximation in these studies was to set Brunt-Väisälä frequency to a constant or a step function over the computational domain. Moreover the values of two physical parameters which appear in this equation were set to zero. (These parameters control the stratification and dispersive effects of the atmosphere - see Sect. 2.) In this (singular) limit the nonlinear terms and one of the leading second order derivatives in the equation drop out and the equation reduces to that of a linear harmonic oscillator over two dimensional domain. Careful studies (Lily, 1979) showed that these approximations set strong limitations on the validity of the derived solutions (Peltier, 1983).

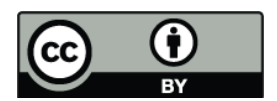

Correspondence to: M. Humi (mhumi@wpi.edu)
Long's equation also provides the theoretical framework for the analysis of experimental data (Shutts, 1988, 1994; Jumper, 2005) under the assumption of shearless base flow. (An assumption which, in general, is not supported by the data; Humi, 2004b.) An extensive list of references appears in (Baines, 1995; Carmen, 2002; Yih, 1980).

An analytic approach to the study of the solutions of this nonlinear equation was initiated recently by the current author (Humi, 2004a, 2006, 2007). We showed that for a base flow without shear and under rather mild restrictions the nonlinear terms in the equation can be simplified. We also identified the "slow variable" that controls the nonlinear oscillations in this equation. Using phase averaging approximation we derived for self similar solutions of this equation a formula for the attenuation of the stream function perturbation with height. This result is generically related to the presence of the nonlinear terms in Long's equation. The impact that shear has on the generation and amplitude of gravity waves was investigated by us in (Humi, 2006). A new representation of this equation in terms of the atmospheric density was derived in (Humi, 2007).

One of the weak aspects of Long's equation is related to the fact that the terrain is represented by the shape of the domain and the boundary conditions. As a result the impact of different terrains on the solution of this equation can only be studied numerically. Furthermore discretization errors which occur in the representation of the terrain render it impractical to consider complex terrain. In part these errors are due to the scale of the terrain relative to the computational domain. Accordingly only simple topographies which were represented by one hill were considered in the literature. Furthermore even for these simple topographies only approximate boundary conditions were applied at the terrain. (See discussion in Sect. 2.)

With this motivation it is our objective in this paper to derive a terrain following formulation of Long's equation in which the terrain is incorporated as part of the coefficients of the differential equation, and the computational domain is always a rectangle. This new representation makes it possible

Published by Copernicus Publications on behalf of the European Geosciences Union and the American Geophysical Union. 
to derive new analytic insights about the solution of this equation in some limiting cases. It will make it easier also to study how the solution varies as a function of the terrain and other parameters that appear in the equation.

The plan of the paper is as follows: Sect. 2 presents a short review of Long's equation and some aspects of its solutions. In Sect. 3 we derive the new formulation of this equation. Section 4 considers Long's equation in some limiting cases of the parameters that govern the solution of this equation. In particular we provide a closed form analytic solution of this equation in the limiting case $\mu=\beta=0$ over general topography. A first order perturbation expansion is developed for the case of "low lying topography" with closed form expression for the stream function in integral form. We end up in Sect. 5 with a summary and conclusions.

\section{Long's equation - a short overview}

In two dimensions $(x, z)$ the flow of a steady inviscid and incompressible stratified fluid is modeled by the following equations:

$$
\begin{aligned}
u_{x}+w_{z} & =0 \\
u \rho_{x}+w \rho_{z} & =0 \\
\rho\left(u u_{x}+w u_{z}\right) & =-p_{x} \\
\rho\left(u w_{x}+w w_{z}\right) & =-p_{z}-\rho g
\end{aligned}
$$

where subscripts indicate differentiation with respect to the indicated variable, $\mathbf{u}=(u, w)$ is the fluid velocity, $\rho$ is its density $p$ is the pressure and $g$ is the acceleration of gravity.

We can non-dimensionalize these equations by introducing

$\bar{x}=\frac{x}{L}, \quad \bar{z}=\frac{N_{0}}{U_{0}} z, \quad \bar{u}=\frac{u}{U_{0}}, \quad \bar{w}=\frac{L N_{0}}{U_{0}^{2}} w$

$\bar{\rho}=\frac{\rho}{\bar{\rho}_{0}}, \quad \bar{p}=\frac{N_{0}}{g U_{0} \bar{\rho}_{0}} p$

where $L$ represents a characteristic horizontal length, and $U_{0}, \overline{\rho_{0}}$ represent respectively the free stream velocity and averaged base density (i.e. here $\bar{\rho}_{0}$ is a constant). $N_{0}^{2}$ is an averaged value of the Brunt-Väisälä frequency

$N^{2}=-\frac{g}{\rho_{0}} \frac{d \rho_{0}}{d z}$

where $\rho_{0}=\rho_{0}(z)$ is the base density.

In these new variables Eqs. (1)-(4) take the following form (for brevity we drop the bars)

$$
\begin{aligned}
u_{x}+w_{z} & =0 \\
u \rho_{x}+w \rho_{z} & =0 \\
\beta \rho\left(u u_{x}+w u_{z}\right) & =-p_{x} \\
\beta \rho\left(u w_{x}+w w_{z}\right) & =-\mu^{-2}\left(p_{z}+\rho\right)
\end{aligned}
$$

where

$$
\begin{aligned}
& \beta=\frac{N_{0} U_{0}}{g} \\
& \mu=\frac{U_{0}}{N_{0} L} .
\end{aligned}
$$

$\beta$ is the Boussinesq parameter (Davis, 1999) (this name has nothing to do with the "Boussinesq approximation") which controls stratification effects (assuming $U_{0} \neq 0$ ) and $\mu$ is the long wave parameter which controls dispersive effects (or the deviation from the hydrostatic approximation). In the limit $\mu=0$ the hydrostatic approximation is fully satisfied (Smith, 1980, 1989).

In view of Eq. (7) we can introduce a stream function $\Psi$ so that

$u=\Psi_{z}, \quad w=-\Psi_{x}$.

Using this stream function we can rewrite Eq. (8) as

$J\{\rho, \Psi\}=0$

where for any two (smooth) functions $f, g$

$J\{f, g\}=\frac{\partial f}{\partial x} \frac{\partial g}{\partial z}-\frac{\partial f}{\partial z} \frac{\partial g}{\partial x}$

Equation (14) implies that the functions $\rho, \Psi$ are dependent on each other and we can express each of them in terms of the other. Thus we can write $\Psi$ as $\Psi(\rho)$ (or $\rho$ as $\rho(\Psi)$; Humi, 2007).

After a long algebra one can derive the following equation for $\Psi$ (Dubreil, 1934; Long, 1953; Davis, 1999)

$\Psi_{z z}+\mu^{2} \Psi_{x x}-N^{2}(\Psi)\left[z+\frac{\beta}{2}\left(\Psi_{z}^{2}+\mu^{2} \Psi_{x}^{2}\right)\right]=S(\Psi)$

where

$N^{2}(\Psi)=-\frac{\rho_{\Psi}}{\beta \rho}$

is the nondimensional Brunt-Väisälä frequency. We observe that in this definition $N^{2}$ is a function of $\Psi$. (As a result it can be an additional source of nonlinearity in Eq. 16.) This is in contrast to the previous definition of this quantity in Eq. (6) which depends only on the base state. In the following we assume without loss of generality that the direction of base flow is from left to right along the $\mathrm{x}$-axis. Furthermore we assume it to be a function of $z$ only.

$S(\Psi)$ is some unknown function which is determined from the base flow. To carry out this determination of $S$ we consider Eq. (16) as $x \rightarrow-\infty$ and express the left hand side of this equation in terms of $\Psi$ only. (Assuming that disturbances do not propagate far upstream; Baines, 1995; Yih, 1980). Equation (16) is referred to as Long's equation.

For example if we let

$\lim _{x \rightarrow-\infty} \Psi(x, z)=z$ 
i.e. consider a shearless base flow with $\lim _{x \rightarrow-\infty} u(x, z)=1$ then

$S(\Psi)=-N^{2}(\Psi)\left(\Psi+\frac{\beta}{2}\right)$

and Eq. (16) becomes:

$\Psi_{z z}+\mu^{2} \Psi_{x x}-N^{2}(\Psi)\left[z-\Psi+\frac{\beta}{2}\left(\Psi_{z}^{2}+\mu^{2} \Psi_{x}^{2}-1\right)\right]=0$.

It is evident from this derivation that different profiles for the base flow as $x \rightarrow-\infty$ will lead to different forms of $S(\Psi)$ (Humi, 2006).

For a general base flow in an unbounded domain over topography with shape $f(x)$ and maximum height $H_{0}$ the following boundary conditions are imposed on $\Psi$

$\lim _{x \rightarrow-\infty} \Psi(x, z)=\Psi_{0}(z)$

$\Psi(x, \tau f(x))=$ constant,$\quad \tau=\frac{H_{0} N_{0}}{U_{0}}$

where the constant in Eq. (22) is (usually) set to zero. As to the boundary condition at $x \rightarrow \infty$ it is appropriate to set

$\lim _{x \rightarrow \infty} \Psi(x, z)=\Psi_{0}(z)$

(in spite of the fact that Long's equation contains no dissipation terms). However over finite computational domain only radiation boundary conditions can be imposed in this limit. Similarly as $z \rightarrow \infty$ it is customary to impose (following Durran, 1992) radiation boundary conditions. (The imposition of these boundary conditions is discussed in detail in Sect. 4.1.)

For the perturbation from the shearless base flow

$\eta=\Psi-z$

Equation (20) becomes

$\eta_{z z}-\alpha^{2} \eta_{z}^{2}+\mu^{2}\left(\eta_{x x}-\alpha^{2} \eta_{x}^{2}\right)-N^{2}(\eta)\left(\beta \eta_{z}-\eta\right)=0$

where

$\alpha^{2}=\frac{N^{2}(\Psi) \beta}{2}$.

We observe that when $|\tau| \ll 1$ the boundary condition Eq. (22) can be approximated by

$\eta(x, 0)=-\tau f(x)$.

When $N$ is constant eq. (24) is invariant with respect to translations in $x, z$ and hence admits self-similar solutions of the form $\eta=f(k x+m z)$ (Humi, 2004a). These solutions are interpreted as gravity waves that are generated by the flow over the topography.

From a numerical point of view it is a common practice (Durran, 1992; Lily, 1979; Davis, 1999) to solve Eq. (24) in the limit $\beta=0$ and $\mu=0$ with constant $N$ over the domain. However observe that the definition of $N$ in Long's equation is given by Eq. (17) and it depends on $\Psi$. In some other numerical simulations the computational domain is divided into subdomains where $N$ is constant in each subdomain but this led to numerical instabilities at the interface between these subdomains.

In these limits Eq. (24) reduces then to a linear equation

$\eta_{z z}+N^{2} \eta=0$.

We observe that the limit $\beta=0$ can be obtained either by letting $U_{0} \rightarrow 0$ or $N_{0} \rightarrow 0$. In the following we assume that this limit is obtained as $U_{0} \rightarrow 0$ (so that stratification persists in this limit and the leading term in $N_{0}$ is not zero).

Equation (27) is a singular limit of Long's equation as one of the leading second order derivatives drops when $\mu=0$ and the nonlinear terms drops out when $\beta=0$ and $N$ is constant. This approximation and its limitations were considered numerically and analytically (Drazin, 1967; Durran, 1992; Humi, 2004a, 2006) and was found to be justified only under strong restrictions even under the assumption that the base flow is shearless. Nevertheless it is used routinely in the actual analysis of atmospheric data (Shutts, 1988; Jumper, 2005; Baines, 1995).

The general solution of Eq. (27) is

$\eta(x, z)=q(x) \cos (N z)+p(x) \sin (N z)$

where the functions $p(x), q(x)$ have to be determined so that the the boundary conditions derived from Eqs. (22), (26) and the radiation boundary conditions are satisfied. These lead in general to an integral equation for $p(x)$ and $q(x)$ and it easy to show (Davis, 1999) that $p(x)=H[q(x)]$ where $H[q(x)]$ is the Hilbert transform of $q(x)$. The boundary condition on the terrain becomes;

$q(x) \cos (\tau N f(x))+H[q(x)] \sin (\tau N f(x))=-\tau f(x)$.

This integral equation has to be solved numerically (Drazin, 1961; Durran, 1992; Davis, 1999; Kar, 1995).

\section{Terrain following formulation}

To derive a terrain following formulation of Long's equation which incorporates the terrain in the coefficients of the differential equation (rather than the shape of the domain) we introduce Gal-Chen transformation. If the height of the (bottom) terrain is described by a sufficiently smooth function $z=h(x)$ and the height of the computational flow region is finite, i.e. $h(x) \leq z \leq H$, where $H$ is a constant, then this transformation is given by

$\bar{x}=x, \quad \bar{z}=H \frac{z-h(x)}{H-h(x)}$.

Under this transformation we have

$\frac{\partial}{\partial x}=\frac{\partial}{\partial \bar{x}}+G^{12} \frac{\partial}{\partial \bar{z}}, \quad \frac{\partial}{\partial z}=\frac{1}{\sqrt{G}} \frac{\partial}{\partial \bar{z}}$ 
where

$\frac{1}{\sqrt{G}}=\frac{H}{H-h(x)}, \quad G^{12}=\frac{1}{\sqrt{G}}\left(\frac{\bar{z}}{H}-1\right) h^{\prime}(x)$.

Furthermore the expression of the Laplace operator becomes

$$
\begin{aligned}
\bar{\nabla}^{2}= & \frac{\partial^{2}}{\partial \bar{x}^{2}}+\left[\frac{1}{G}+\left(G^{12}\right)^{2}\right] \frac{\partial^{2}}{\partial \bar{z}^{2}}+2 G^{12} \frac{\partial^{2}}{\partial \bar{x} \partial \bar{z}}+ \\
& {\left[\frac{\partial G^{12}}{\partial \bar{x}}+G^{12} \frac{\partial G^{12}}{\partial \bar{z}}\right] \frac{\partial}{\partial \bar{z}} . }
\end{aligned}
$$

Under this transformation the continuity Eq. (7) becomes

$\frac{\partial u}{\partial \bar{x}}+G^{12} \frac{\partial u}{\partial \bar{z}}+\frac{1}{\sqrt{G}} \frac{\partial w}{\partial \bar{z}}=0$.

However, if we introduce

$v=\frac{1}{\sqrt{G}}\left(w+\sqrt{G} G^{12} u\right)$

then it is a simple algebra to show that Eq. (34) can be rewritten as

$$
\frac{\partial}{\partial \bar{x}}(\sqrt{G} u)+\frac{\partial}{\partial \bar{z}}(\sqrt{G} v)=0
$$

From this equation we see that we can introduce a "terrain following stream function" $\psi$ so that

$\bar{u}=\sqrt{G} u=\frac{\partial \psi}{\partial \bar{z}}, \quad \bar{v}=\sqrt{G} v=-\frac{\partial \psi}{\partial \bar{x}}$.

Multiplying Eq. (8) by $\sqrt{G}$ we can rewrite this equation in the following form:

$\bar{u} \frac{\partial \rho}{\partial \bar{x}}+\bar{v} \frac{\partial \rho}{\partial \bar{z}}=0$.

Using Eq. (37) this can be rewritten as

$\bar{J}\{\rho, \psi\}=0$

where $\bar{J}$ is defined as in Eq. (15) but with differentiations with respect to $(\bar{x}, \bar{z})$. Equation (39) implies that $\rho(\bar{x}, \bar{z})=\rho(\psi(\bar{x}, \bar{z}))$ (and vice versa).

To eliminate the pressure term from Eqs. (9) and (10) we differentiate Eq. (9) by $z$ and apply the operator $\mu^{2} \frac{\partial}{\partial x}$ to Eq. (10) and subtract. We obtain

$\beta \mu^{2} \rho_{x}\left(u w_{x}+w w_{z}\right)-\beta \rho_{z}\left(u u_{x}+w u_{z}\right)+$

$\beta \mu^{2} \rho\left(u w_{x}+w w_{z}\right)_{x}-\beta \rho\left(u u_{x}+w u_{z}\right)_{z}=-\rho_{x}$.

Using Eq. (8) the first two terms in this equation can be written as

$\beta \mu^{2} \rho_{x}\left(u w_{x}+w w_{z}\right)-\beta \rho_{z}\left(u u_{x}+w u_{z}\right)=\beta\left[\mu^{2}\left(-\rho_{z} w w_{x}+\right.\right.$

$\left.\left.\rho_{x} w w_{z}\right)-\rho_{z} u u_{x}+\rho_{x} u u_{z}\right]=\frac{\beta}{2}\left[\rho_{x}\left(u^{2}+\mu^{2} w^{2}\right)_{z}-\right.$

$\left.\rho_{z}\left(u^{2}+\mu^{2} w^{2}\right)_{x}\right]=\frac{\beta}{2 \sqrt{G}}\left[\rho_{\bar{x}}\left(u^{2}+\mu^{2} w^{2}\right)_{\bar{z}}-\right.$

$\left.\rho_{\bar{z}}\left(u^{2}+\mu^{2} w^{2}\right)_{\bar{x}}\right]=\frac{\beta}{2 \sqrt{G}} \bar{J}\left\{\rho, u^{2}+\mu^{2} w^{2}\right\}$.
Using Eqs (35) and (37) to re-express $u^{2}+\mu^{2} w^{2}$ we have

$\frac{\beta}{2 \sqrt{G}} \bar{J}\left\{\rho, u^{2}+\mu^{2} w^{2}\right\}=\frac{\beta}{2 \sqrt{G}} \rho_{\psi} \bar{J}\left\{\psi, \mu^{2}\left(\psi_{\bar{x}}\right)^{2}+\right.$

$\left.2 \mu^{2} G^{12} \psi_{\bar{x}} \psi_{\bar{z}}+\left[\frac{1}{G}+\mu^{2}\left(G^{12}\right)^{2}\right]\left(\psi_{\bar{z}}\right)^{2}\right\}$

The third and the fourth terms in Eq. (40) can be rewritten using Eq. (7) as

$\beta \mu^{2} \rho\left(u w_{x}+w w_{z}\right)_{x}-\beta \rho\left(u u_{x}+w u_{z}\right)_{z}=\beta \rho\left[u\left(\mu^{2} w_{x}-u_{z}\right)\right.$

$\left.+v\left(\mu^{2} w_{x}-u_{z}\right)\right]=-\frac{\beta \rho}{\sqrt{G}} \bar{J}\{\psi, \chi\}$

where $\chi=\mu^{2} w_{x}-u_{z}$ is the vorticity. Expressing $\chi$ in terms of $\psi$ we have

$\chi=-\bar{\nabla}_{\mu}^{2} \psi$

where

$$
\begin{aligned}
\bar{\nabla}_{\mu}^{2}= & \mu^{2}\left\{\frac{\partial^{2}}{\partial \bar{x}^{2}}+2 G^{12} \frac{\partial^{2}}{\partial \bar{x} \partial \bar{z}}+\left[\frac{\partial G^{12}}{\partial \bar{x}}+G^{12} \frac{\partial G^{12}}{\partial \bar{z}}\right] \frac{\partial}{\partial \bar{z}}\right\}+ \\
& {\left[\frac{1}{G}+\mu^{2}\left(G^{12}\right)^{2}\right] \frac{\partial^{2}}{\partial \bar{z}^{2}} }
\end{aligned}
$$

is the "terrain following Laplace operator".

Finally for the right hand side of Eq. (40) we have

$-\rho_{x}=-\frac{1}{\sqrt{G}} \bar{J}\{\rho, g\}=-\frac{\rho_{\psi}}{\sqrt{G}} \bar{J}\{\psi, g\}$

where

$g(\bar{x}, \bar{z})=\bar{z}+h(\bar{x})\left(1-\frac{\bar{z}}{H}\right)$

Combining all the results contained in Eqs. (41)-(46) we can re-express Eq. (40) in the following form:

$\bar{J}\left\{\psi, \bar{\nabla}_{\mu}^{2} \psi-\frac{N^{2}(\psi) \beta}{2}\left[\mu^{2}\left(\psi_{\bar{x}}\right)^{2}+2 \mu^{2} G^{12} \psi_{\bar{x}} \psi_{\bar{z}}+\right.\right.$

$\left.\left.\left(\frac{1}{G}+\mu^{2}\left(G^{12}\right)^{2}\right)\left(\psi_{\bar{z}}\right)^{2}\right]-N^{2}(\psi) g(\bar{x}, \bar{z})\right\}=0$

where $N^{2}(\psi)$ is defined as in Eq. (17). Hence it follows that, $\bar{\nabla}_{\mu}^{2} \psi-\frac{N^{2}(\psi) \beta}{2}\left[\mu^{2}\left(\psi_{\bar{x}}\right)^{2}+2 \mu^{2} G^{12} \psi_{\bar{x}} \psi_{\bar{z}}+\right.$

$\left.\left(\frac{1}{G}+\mu^{2}\left(G^{12}\right)^{2}\right)\left(\psi_{\bar{z}}\right)^{2}\right]-N^{2}(\psi) g(\bar{x}, \bar{z})=S(\psi)$.

This is the terrain following form of Long's equation. At this juncture it might be asked why one can not "save" this derivation and apply the terrain following transformation (30) directly to (16). Doing so will yield an extremely complicated 
equation. This has been avoided in our derivation by the use of the "terrain following stream function" in Eq. (37).

To determine the function $S(\psi)$ in Eq. (48) we assume that

$\lim _{\bar{x} \rightarrow-\infty} h(\bar{x})=0$

and that (as an example) $\psi$ satisfies

$\lim _{x \rightarrow-\infty} \psi(\bar{x}, \bar{z})=\bar{z}$

It follows then that

$S(\psi)=-N^{2}(\psi)\left(\psi+\frac{\beta}{2}\right)$

and Long's equation becomes:

$\bar{\nabla}_{\mu}^{2} \psi-\frac{N^{2}(\psi) \beta}{2}\left[\mu^{2}\left(\psi_{\bar{x}}\right)^{2}+2 \mu^{2} G^{12} \psi_{\bar{x}} \psi_{\bar{z}}+\right.$
$\left.\left(\frac{1}{G}+\mu^{2}\left(G^{12}\right)^{2}\right)\left(\psi_{\bar{z}}\right)^{2}\right]-N^{2}(\psi)\left[g(\bar{x}, \bar{z})-\psi-\frac{\beta}{2}\right]=0$.

In this representation the flow domain is a rectangle $[a, b] \times[0, H]$ or an infinite stripe $[-\infty, \infty] \times[0, H]$. The boundary condition at the bottom topography is

$\mathbf{u} \cdot \mathbf{n}=0$

where $\mathbf{n}$ is the normal to the topography which is described by the curve $h(x)$. Hence this normal is given by $\mathbf{n}=\left(-h^{\prime}(x), 1\right)$. Using Eqs. (35) and (37) this leads to the boundary condition

$\psi(\bar{x}, 0)=$ constant

and this constant can be chosen to be zero. The other boundary condition that has to be imposed on $\psi$ is a radiation boundary condition at $\bar{z}=H$ (which implies that the outgoing wave is not reflected by the boundary).

To obtain an equation for the perturbation from the base state we set

$\psi(\bar{x}, \bar{z})=\bar{z}+\eta(\bar{x}, \bar{z})$

Substituting this in Eq. (51) we obtain the following (exact) equation for $\eta$

$\bar{\nabla}_{\mu}^{2} \eta+N^{2}(\eta) \eta-\frac{N^{2}(\eta) \beta}{2}\left[\mu^{2}\left(\eta_{\bar{x}}\right)^{2}+2 \mu^{2} G^{12} \eta_{\bar{x}}\left(\eta_{\bar{z}}+1\right)+\right.$

$\left.\left(\frac{1}{G}+\mu^{2}\left(G^{12}\right)^{2}\right)\left[\left(\eta_{\bar{z}}\right)^{2}+2 \eta_{\bar{z}}\right]\right]=$

$-\mu^{2}\left(\frac{\partial G^{12}}{\partial \bar{x}}+G^{12} \frac{\partial G^{12}}{\partial \bar{z}}\right)+N^{2}(\eta)\left\{h(\bar{x})\left(1-\frac{\bar{z}}{H}\right)+\right.$

$\left.\frac{\beta}{2}\left[\left(\frac{1}{G}+\mu^{2}\left(G^{12}\right)^{2}\right)-1\right]\right\}$.

\section{Analytic solutions of Long's equation}

In the traditional representation of Long's equation the topography determines the shape of the flow domain and as a result it is not feasible to obtain analytic solutions to this equation even in some limits of the parameters $\beta$ and $\mu$. We now show that this problem can be overcome in some limiting cases when the terrain following formulation of this equation is used.

We consider two limiting cases $\beta=0, \mu=0$ and $\beta \neq 0, \mu=0$ we also assume $N^{2}(\psi)=$ constant. For brevity we drop in the following the bars over $x, z$.

\subsection{The limiting case $\beta=0, \mu=0$}

In this case Eq. (51) simplifies to

$\frac{\partial^{2} \psi}{\partial z^{2}}+G N^{2} \psi=G N^{2}\left[z+h(x)\left(1-\frac{z}{H}\right)\right]$

whose general solution is

$\psi=A(x) \cos (v z)+B(x) \sin (v z)+\left[z+h(x)\left(1-\frac{z}{H}\right)\right]$.

Here $v=N \sqrt{G}$ and $A(x), B(x)$ are functions which have to be determined from the boundary conditions.

The boundary condition (52) implies $A(x)=-h(x)$. To determine $B(x)$ we must apply the radiation boundary condition as $z \rightarrow \infty$ on the solution. To this end we must insure that the vertical group velocity of the wave is positive. Using the dispersion relation for hydrostatic flow given in (Baines, 1995 , p. 181) this group velocity is:

$c_{g}=\frac{N k \operatorname{sgn}(v)}{v^{2}}$

where $k$ is the horizontal wave number. We deduce then that the vertical group velocity is positive when $k v \geq 0$.

To impose this condition on the solution (56) we express $A(x), B(x)$ in Fourier integral form

$A(x)=\int_{-\infty}^{\infty} a(k) e^{i k x} d k, \quad B(x)=\int_{-\infty}^{\infty} b(k) e^{i k x} d k$

where $k$ is the horizontal wave number. We deduce then that the solution (56) can be written as

$$
\begin{aligned}
\psi= & \frac{1}{2}\left\{\int_{-\infty}^{\infty}(a(k)-i b(k)) e^{i(k x+v z)} d k+\int_{-\infty}^{\infty}(a(k)+\right. \\
& \left.i b(k)) e^{i(k x-v z)} d k\right\}+\left[z+h(x)\left(1-\frac{z}{H}\right)\right]
\end{aligned}
$$

To satisfy the radiation boundary condition for $z \rightarrow \infty$ the first and second integral must vanish for $k<0$ and $k>0$, respectively. Therefore $a(k)$ and $b(k)$ must satisfy

$a(k)=-i \operatorname{sgn}(k) b(k)$ 
which implies that $B(x)$ is the Hilbert transform of $A(x)=-h(x)$ i.e.

$B(y)=-H(h(x))=-\frac{1}{\pi} \mathrm{P} . \mathrm{V} . \int_{-\infty}^{\infty} \frac{h(x)}{x-y} d x$.

This represents a complete analytic solution of Long's equation for this limiting case.

In particular if

$h_{1}(x)=\frac{1}{\left(1+x^{2}\right)^{3 / 2}}$

then

$B_{1}(x)=\frac{2}{\pi}\left[\frac{x}{1+x^{2}}+\frac{a \sinh x}{\left(1+x^{2}\right)^{3 / 2}}\right]$.

Similarly if $h(x)$ is given by a "witch of Agnesi" curve

$h_{2}(x)=\frac{a^{2}}{\left(a^{2}+x^{2}\right)}$

then

$B_{2}(x)=-\frac{a x}{a^{2}+x^{2}}$.

Since the Hilbert transform is linear one can use these results to compute the stream function (in this limit of the parameters) over any terrain that is composed of $h(t)$ which the sum (up to translations) of the height functions given above or any others for which the Hilbert transform can be computed analytically. For example if

$h(x)=\frac{c_{1}}{\left(1+x^{2}\right)^{3 / 2}}+\frac{c_{2}}{\left(1+(x-5)^{2}\right)^{3 / 2}}+\frac{c_{3} a^{2}}{\left(a^{2}+(x+5)^{2}\right)}$

(where $c_{i}, i=1,2,3$ are constants) then

$B(x)=c_{1} B_{1}(x)+c_{2} B_{1}(x-5)+c_{3} B_{2}(x+5)$

It should be noted however that in these expressions $\psi$ represents the "terrain following stream function" which was defined in (37). We can recover the flow field $(u, w)$ from this function using (37) and (35). From this vector field it is easy to compute the "regular" stream function $\phi(x, z)$. Figures 1 and 2 depict the regular stream functions for the terrains given by (62) and (66) respectively. These figures were obtained by direct evaluation of the formulas given above.

We compare now these analytic results with the solution methodology that has been used previously in the literature as was discussed Sect. 2. First we note that this analytic solution requires only the direct (and simple) computation of the Hilbert transform of the terrain function $h(x)$. This is a straightforward procedure even if it has to be done numerically. On the other hand to compute $q(x)$ using (29) requires in general the solution of an integral equation. To do so one must use an iterative algorithm which might turn out to be unstable or non-convergent over complex terrain. Furthermore there is the issue of applying the boundary conditions on $\psi$

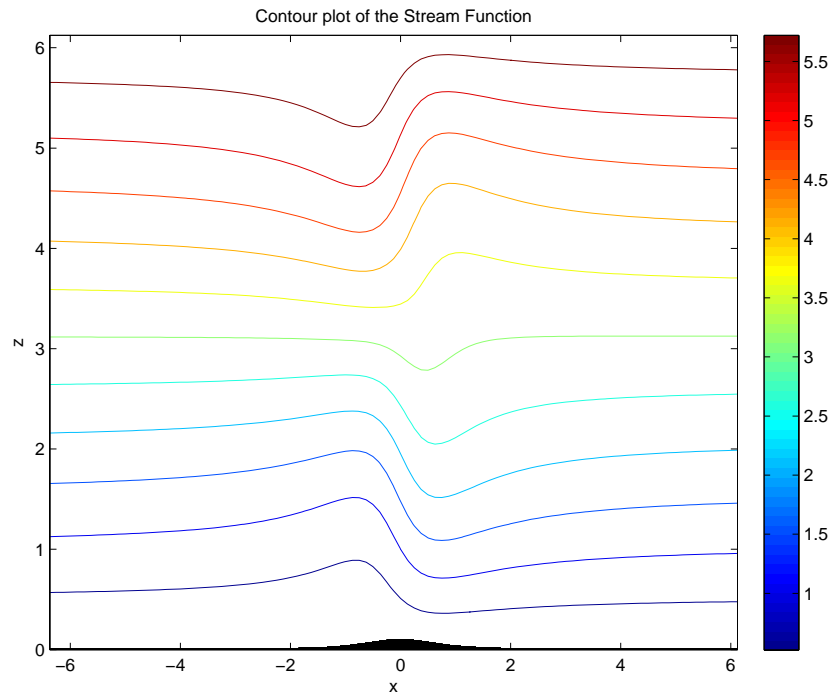

Fig. 1. The regular stream function $\phi$ over one hill centered at $x=0$ with height $h(x)=\epsilon h_{1}(x)$ where $h_{1}$ is given by Eq. (62), $\epsilon=0.1$, $N=1, \beta=0, \mu=0$.

at the terrain. To this end the procedure discussed in Sect. 2 requires the use of the approximations that lead to (26). As a result the equation that is used to compute $q(x)(\mathrm{Eq} .29)$ is also an approximate equation which will yield at best approximate solution for this function. On the other hand the application of the boundary conditions using the procedure discussed in this section is exact and does not place constraints on the height of the terrain.

From an experimental geophysical point of view it has been a common practice to assume that the gravity wave generated by a flow over terrain is of the form $\sin (k x+m z)$ (or similar) (Shutts, 1988; Jumper, 2005; Eckermann, 1999; Dewan, 1998). This has led to difficulties in the eduction of this wave from experimental data. Our results show that this form of the wave is incorrect (at least in principle). Furthermore as Fig. 2 demonstrates complex terrain can alter drastically the shape and amplitude of this wave due to interference effects.

\subsection{The limiting case $|\boldsymbol{h}(\boldsymbol{x})| \ll 1, \boldsymbol{\beta} \ll 1$}

Under these limiting conditions it is appropriate to introduce an order parameter $\epsilon$ so that

$h(x)=\epsilon h_{1}(x)$

and consider a two parameter expansion of the stream function in $\epsilon$ and $\beta$ viz.

$\psi(x, z)=\psi^{(0)}(x, z)+\beta \psi^{(1)}(x, z)+\epsilon \psi^{(2)}(x, z)+O\left(\epsilon^{2}, \beta^{2}, \epsilon \beta\right)$

The first order expansion of (51) in the parameters $\epsilon$ and $\beta$ yields the following equations for $\psi^{i}, i=0,1,2$.

$\mu^{2} \psi_{x x}^{(0)}+\psi_{z z}^{(0)}+N^{2} \psi^{(0)}=N^{2} z$ 
$\mu^{2} \psi_{x x}^{(1)}+\psi_{z z}^{(1)}+N^{2} \psi^{(1)}-\frac{1}{2} \mu^{2} N^{2}\left(\psi_{x}^{(0)}\right)^{2}=0$

$\mu^{2} \psi_{x x}^{(2)}+\psi_{z z}^{(2)}+N^{2} \psi^{(2)}=\left(1-\frac{z}{H}\right)$

$\left[N^{2} h_{1}+\mu^{2}\left(h_{1}^{\prime \prime} \psi_{z}^{(0)}+2 h_{1}^{\prime} \psi_{x z}^{(0)}\right)\right]-\frac{2 h_{1}}{H} \psi_{z z}^{(0)}$

It is easy to see that the solution to (70) subject to the the boundary conditions (49), (52) is

$\psi^{(0)}(x, z)=z$.

Due to this result (71) (72) simplify and it is straight forward to see that the general solution to these equations is

$\psi^{(1)}(x, z)=$

$\int_{0}^{N / \mu} \sin \lambda z\left[A_{1}(\omega) \cos (\omega x)+B_{1}(\omega) \sin (\omega x)\right] d \omega+$

$\int_{0}^{N / \mu} \cos \lambda z\left[C_{1}(\omega) \cos (\omega x)+D_{1}(\omega) \sin (\omega x)\right] d \omega$,

where $\mu^{2} \omega^{2}+\lambda^{2}=N^{2}$. Similarly,

$\psi^{(2)}(x, z)=$

$\int_{0}^{N / \mu} \sin \lambda z\left[A_{2}(\omega) \cos (\omega x)+B_{2}(\omega) \sin (\omega x)\right] d \omega+$

$\int_{0}^{N / \mu} \cos \lambda z\left[C_{2}(\omega) \cos (\omega x)+D_{2}(\omega) \sin (\omega x)\right] d \omega+\frac{H-z}{H} h_{1}(x)$.

Applying the boundary condition $(52)$ to the $\psi^{(1)}(x, z)$ and $\psi^{(2)}(x, z)$ we infer that $C_{1}=D_{1}=0$ and

$\int_{0}^{N / \mu}\left[C_{2}(\omega) \cos (\omega x)+D_{2}(\omega) \sin (\omega x)\right] d \omega=-h_{1}(x)$.

The radiation boundary condition implies that

$\int_{0}^{N / \mu}\left[A_{2}(\omega) \cos (\omega x)+B_{2}(\omega) \sin (\omega x)\right] d \omega=-H\left(h_{1}(x)\right)$

and $A_{1}=B_{1}=0$. Thus, in the present settings, the first order contribution of the $\beta$ terms vanishes when the base stream function satisfies (49). This can be verified directly by performing a one parameter perturbation expansion on $\psi$ viz. by letting $\beta=\epsilon \beta_{1}$ and

$\psi(x, z)=\psi^{(0)}(x, z)+\epsilon \psi^{(1)}(x, z)+O\left(\epsilon^{2}\right)$

When the topography is given by Eq. (64) with $a=1$ it is easy to show using Eq. (76) that $D_{2}=0$ and $C_{2}(\omega)=-e^{-\omega}$. Similarly (using Eq. 65) we obtain $A_{2}=0$ and $B_{2}(\omega)=-e^{-\omega}$. Substituting this data in Eq. (74) we can compute $\psi^{(2)}$ by numerical integration. We observe however that these integrals are highly oscillatory and appropriate numerical routines have to be used to evaluate them accurately. The results of these computations with $\mu=0.25$ (re-expressed in terms of the regular stream function $\phi$ ) are shown in Fig. 3.

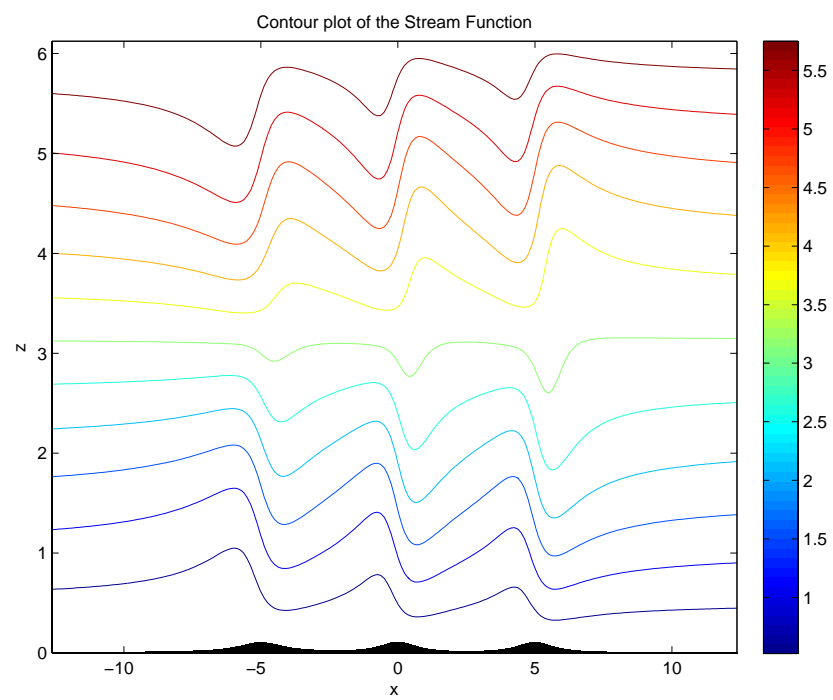

Fig. 2. Same as Fig. 1 but $h(x)$ is given by Eq. (66) with $c_{1}=c_{2}=c_{3}=1$ and $a=1$.

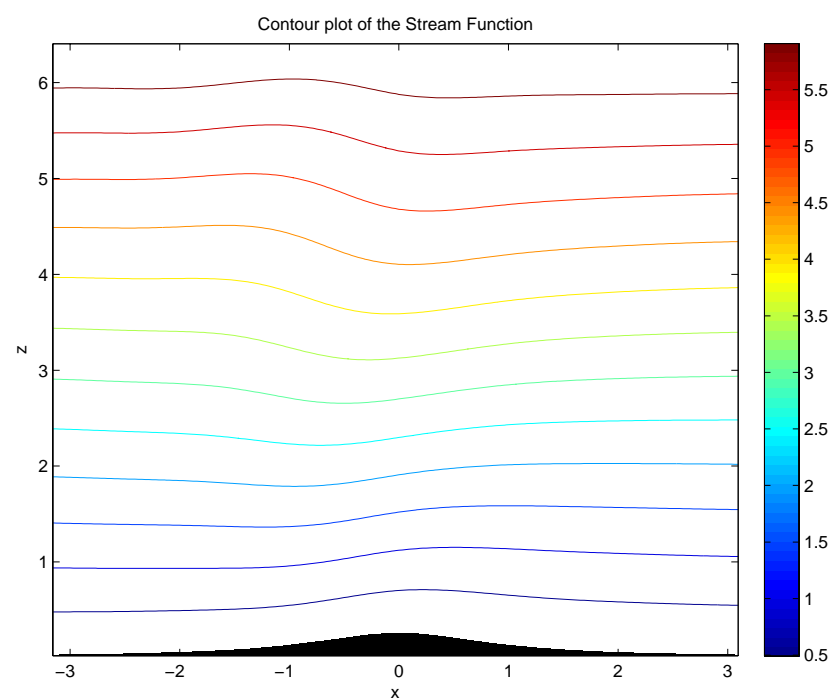

Fig. 3. The regular stream function $\phi$ over one hill centered at $x=0$ with height $h(x)=\epsilon h_{1}(x)$ where $h_{1}$ is given by Eq. (64) with $a=1, \epsilon=0.25, N=1, \beta=0, \mu=0.25$. The computations are based on Eqs. (73) and (74).

\subsection{The limiting case $\beta \neq 0, \mu=0$}

In this limiting case Eq. (51) becomes

$$
\frac{\partial^{2} \psi}{\partial z^{2}}-G N^{2}\left\{-\psi+\frac{\beta}{2}\left[\frac{1}{G}\left(\frac{\partial \psi}{\partial z}\right)^{2}-1\right]+z+h(x)\left(1-\frac{z}{H}\right)\right\}=0 .
$$

Since this is a nonlinear equation we can find an approximate analytical solution using first order perturbation expansion under the assumption that $\alpha^{2}=\frac{N^{2} \beta}{2} \ll 1$ (which is sat- 


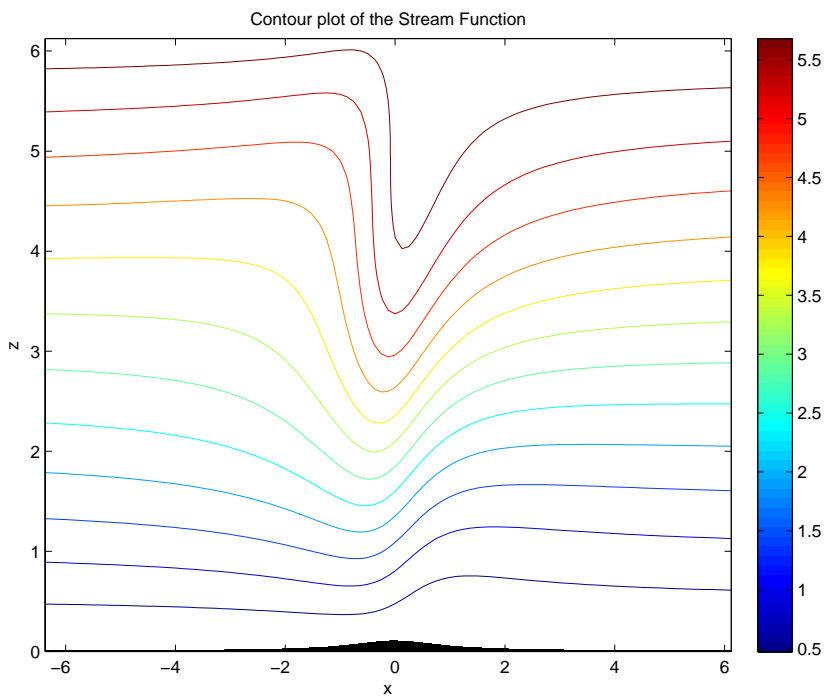

Fig. 4. The regular stream function $\phi$ over one hill centered at $x=0$ with height $h(x)=\epsilon h_{1}(x)$ where $h_{1}$ is given by Eq. (64) with $a=1$, $\epsilon=0.1, \alpha^{2}=0.01, \mu=0$. The computations are based on Eqs. (82)(87).

isfied in most practical situations). Expressing $\psi$ approximately as

$\psi=\psi_{0}+\alpha^{2} \psi_{1}$

and substituting this expression in Eq. (61) we obtain to order zero and one in the parameter $\alpha^{2}$ the following equations

$\frac{\partial^{2} \psi_{0}}{\partial z^{2}}+G N^{2} \psi_{0}=G N^{2}\left[z+h(x)\left(1-\frac{z}{H}\right)\right]$

$\frac{\partial^{2} \psi_{1}}{\partial z^{2}}+G N^{2} \psi_{1}-\left(\frac{\partial \psi_{0}}{\partial z}\right)^{2}=-G$

The boundary conditions on $\psi_{0}, \psi_{1}$ are given by Eq. (52) at $z=0$ and radiation boundary conditions as $z \rightarrow \infty$.

Solving these (linear) equations for $\psi_{0}$ and $\psi_{1}$ we obtain the following expressions for their solutions

$$
\begin{aligned}
& \psi_{0}(x, z)=A(x) \cos (v z)+B(x) \sin (v z)+\left[z+h(x)\left(1-\frac{z}{H}\right)\right] \\
& \psi_{1}(x, z)=C_{1}(x) \cos (v z)+C_{2}(x) \sin (v z)+f_{1}(x, z)+f_{2}(x, z)+f_{3}(x)
\end{aligned}
$$

where

$$
\begin{aligned}
& f_{1}(x, z)=\frac{\left(A^{2}(x)-B^{2}(x)\right) \cos (2 v z)+2 A(x) B(x) \sin (2 v z)}{6} \\
& f_{2}(x, z)=\frac{(H-h(x))((v A(x) z+B(x)) \cos (v z)+v B(x) z \sin (v z))}{H v} \\
& f_{3}(x)=\frac{A(x)^{2}+B(x)^{2}}{2}
\end{aligned}
$$

where Eq. (32) was used to simplify Eq. (86).
Since $\mathrm{V}(80)$ is the same as $\mathrm{V}(55)$ it follows that $A(x)=-h(x)$ and $B(x)$ is given by Eq. (61). Applying the boundary condition Eq. (52) to $\psi_{1}$ we obtain

$$
C_{1}(x)=-\frac{1}{3}\left(2 A(x)^{2}+B(x)^{2}\right)-\frac{1}{v}\left(1-\frac{h(x)}{H}\right) B(x)
$$

Similarly the radiation boundary condition leads to $C_{2}(y)=-H\left(C_{1}(x)\right)$. For a topography described by Eq. (64) with $a=1$ we computed $C_{1}$ and $C_{2}$ (analytically) and used (83) to calculate $\psi_{1}$. Figure 4 displays the corresponding regular stream function $\phi(x, z)$ when $\alpha^{2}=10^{-3}$.

\section{Summary and conclusions}

We derived in this paper a terrain following formulation of Long's equation in which the topography is "absorbed" in the coefficients of the differential equation representing the flow rather than being part of the boundary conditions. We used this representation to solve Long's equation analytically in some limiting cases and over complex topography. The new formulation also opens the possibility to develop analytical estimates which compare the solutions of this equation over different topographies. The analytical and numerical treatment of the solutions to Eq. (52) for general values of $\mu$ and $\beta$ will be left to a subsequent publication.

From a geophysical point of view it well known that some present models for the generation of gravity waves over estimate this effect (Eckermann, 1999; Dewan, 1998; Humi, 2004b). Partially, this is due to the fact that they use oversimplified representation of the terrain. Furthermore they do not take into account the effects that are due to complex terrain (as demonstrated by our simulations). We believe that the new form of Long's equation will make it easier to consider more realistic representations of the terrain and its effect on the generation and propagation of gravity waves.

Edited by: R. Grimshaw

Reviewed by: two anonymous referees

\section{References}

Baines, P. G.: Topographic effects in Stratified flows, Cambridge Univ. Press, New-York, 1995.

Nappo, C. J.: Atmospheric Gravity Waves, Academic Press, Boston, 2002.

Davis, K. S.: Flow of Nonuniformly Stratified Fluid of Large Depth over Topography, M.Sc. thesis in Mechanical Engineering, MIT, Cambridge, MA, 1999.

Dewan, E. M., Picard, R. H., O’Neil, R. R., Gardiner, H. A., Gibson, J., Mill, J. D., Richards, E., Kendra, M., and Gallery, W. O.: MSX satellite observations of thunderstorm-generated gravity waves in mid-wave infrared images of the upper stratosphere, Geophys. Res. Lett., 25, 939-942, 1998. 
Drazin, P. G.: On the steady flow of a fluid of variable density past an obstacle, Tellus, 13, 239-251, 1961.

Drazin, P. G. and Moore D. W.: Steady two dimensional flow of fluid of variable density over an obstacle, J. Fluid. Mech., 28, 353-370, 1967.

Dubreil-Jacotin, M. L.: Sur la determination rigoureuse des ondes permanentes periodiques d'ampleur finie, J. Math. Pure. Appl., 13, 217-291, 1934.

Durran, D. R.: Two-Layer solutions to Long's equation for vertically propagating mountain waves, Q. J. Roy. Meteor. Soc., 118, 415-433, 1992.

Eckermann, S. D. and Preusse, P.: Global measurements of stratospheric mountain waves from space, Science, 286, 1534-1537, 1999.

Haagenson, P. L., Dudhia, J., Grell, G. A., and Stauffer, D. R.: The Penn State/NCAR mesoscale model(MM5) source code documentation, NCAR Technical Note, NCAR/TN-392+STR, 1994.

Humi, M.: On the Solution of Long's Equation Over Terrain, Il Nuovo Cimento C, 27, 219-229, 2004a.

Humi, M.: Estimation of Atmospheric Structure Constants from Airplane Data, J. Atmos. Ocean. Tech., 21, 495-500, 2004b.

Humi, M.: On the Solution of Long's Equation with Shear, Siam J. Appl. Math, 66(6), 1839-1852, 2006.

Humi, M.: Density representation of Long's equation, Nonlin. Processes Geophys., 14, 273-283, 2007,

http://www.nonlin-processes-geophys.net/14/273/2007/.

Jumper, G. Y., Vernin, J., Azouit, M., and Trinquet, H.: Comparison of Recent Measurements of Atmospheric Optical Turbulence, AIAA paper, AIAA-2005-4778, 2005.

Kar, S. K. and Turco, R. P.: Formulation of a Lateral Sponge Layer for Limited Area Shallow-Water Models and an Extension for the Vertically Stratified Case, Mon. Weather Rev., 123, 1542-1559, 1995.
Lily, D. K. and Klemp, J. B.: The effect of terrain shape on nonlinear hydrostatic mountain waves, J. Fluid Mech., 95, 241-261, 1979.

Long, R. R.: Some aspects of the flow of stratified fluids I. Theoretical investigation, Tellus, 5, 42-57, 1953.

Long, R. R.: Some aspects of the flow of stratified fluids II. Theoretical investigation, Tellus, 6, 97-115, 1954.

Long, R. R.: Some aspects of the flow of stratified fluids III. Continuous density gradients, Tellus, 7, 341-357, 1955.

Long, R. R.: The Motion of Fluids with Density Stratification, J. Geophys. Res., 64(12), 2151-2163, 1959.

Peltier, W. R. and Clark, T. L.: Nonlinear mountain waves in two and three spatial dimensions, Q. J. Roy. Meteor. Soc., 109, 527548, 1983.

Shutts, G. J., Kitchen, M., and Hoare, P. H.: A large amplitude gravity wave in the lower stratosphere detected by radiosonde, Q. J. Roy. Meteor. Soc., 114, 579-594, 1988.

Shutts, G. J., Healey, P., and Mobbs, S. D.: A multiple sounding technique for the study of gravity waves, Q. J. Roy. Meteor. Soc., 120, 59-77, 1994.

Smith, R. B.: Linear theory of stratified hydrostatic flow past an isolated mountain, Tellus, 32, 348-364, 1980.

Smith, R. B.: Hydrostatic airflow over mountains, Adv. Geophys., 31, 1-41, 1989.

Yih, C.-S.: Equations governing steady two-dimensional large amplitude motion of a stratified fluid, J. Fluid Mech., 29, 539-544, 1967.

Yih, C.-S.: Stratified flows, Academic Press, New York, NY, 1980. 